\title{
Ships Magnetic Anomaly Computation With Integral Equation and Fast Multipole Method
}

\author{
T.-S. Nguyen, J.-M. Guichon, O. Chadebec, P. Labie, and J.-L. Coulomb \\ Grenoble Electrical Eng. Lab., University of Grenoble, Grenoble-INP, Université Joseph Fourier, UMR CNRS 5269, France
}

\begin{abstract}
This paper presents a coupling between integral volume equation method (IVEM) and a fast multipole algorithm (FMM) in order to model ships magnetic anomaly. complex geometries of real vessels associated with dense meshes can be treated in order to compute the magnetic field created by induced magnetization of the hull and coils degaussing systems. Our algorithm has been validated with measurements made on a real submarine mock-up.
\end{abstract}

Index Terms-Fast solvers, integrodifferential equations, magnetostatics, marine technology.

\section{INTRODUCTION}

A NAVAL vessel, magnetized in the earth's magnetic field, creates a local magnetic perturbation. To decrease the possibility of the vessel's detection or its destruction by magnetically fired weapons, it is necessary to minimize this magnetic signature. Degaussing coils are the best known method to reduce this anomaly. The aim of such electrical systems is to create an outside opposite magnetic field by adjusting currents in the coils. To design an optimal degaussing system (which takes in account their number of coils, position, shape and Amp.turns), we need to compute the magnetization and the associated magnetic anomaly of the ship before building. This allows the simultaneous development of the ship and its degaussing system. Until now, the most used numerical method was the finite element method. Many papers have already been published about the computation of the magnetization [1], [2] and the coils effects [3].

However, the modelling of ships magnetic anomaly with finite element method leads to several difficulties. The first is the necessity to mesh volume regions. In numerical modelling, common hypothesis is that the vessel is surrounded by an infinite air region. To compute accurately the field outside involves a very high mesh density far from it. Moreover, domain truncation with boundary conditions can lead to inaccuracies. To solve this problem, some efficient numerical tools have been proposed like the "infinite box" for instance [1]. Another difficulty is the very high ratio between the length of the ship and the thickness of the hull which leads to an unacceptable number of elements if the hull is meshed in its volume. Of course, this situation can be avoided if surface elements, modelling the special magnetic behavior of the sheet, are used [4]. The last but not the least is to model degaussing coils effects. In fact, the coils are located very close from the hull (a few centimeters) and their local magnetic effects are important. An accurate modelling needs the local refinement of the hull's mesh close to the non-meshed inductor coils and then in the surrounding air region. The generation of such meshes is uneasy and CPU costly. Thus, if we consider that a standard ship owns tens of coils, the modelling becomes

Manuscript received May 31, 2010; accepted October 26, 2010. Date of current version April 22, 2011. Corresponding author: O. Chadebec (e-mail: olivier. chadebec@g2elab.grenoble-inp.fr).

Color versions of one or more of the figures in this paper are available online at http://ieeexplore.ieee.org.

Digital Object Identifier 10.1109/TMAG.2010.2091626 quickly too memory consuming. A solution proposed in [3] is to complete the finite element method with to an analytical behavior of the local effect. However this solution remains difficult to generalize and suffers of inaccuracies for real complex geometries.

In this paper, we prefer to use a numerical technique based on the resolution of an integral volume equation. This method is already well-known for its efficiency to solve such problem and has been mainly used recently to solve the inverse problem, meaning the identification of the magnetization starting from magnetic measurements around the hull [5]-[7]. The main advantage is that no mesh of the air region is needed so the method ensures a really good ability to compute accurately stray magnetic field. Moreover, the difficulty of the mesh generation around inductor coils disappears.

However, the approach is strongly limited to compute forward problem meaning starting from the real geometry of a ship and from the material properties to compute the magnetic anomaly. This limitation is due to the necessity to store a fully dense matrix inherent to the use of such numerical method. To solve this problem, we couple our integral formulation with an adaptive multi-level fast multipole method (AMLFMM) to speed-up interactions' computation and to reduce memory requirements.

It must be pointed out that this kind of coupling between integral volume equation and FMM has already been applied recently to model classical electrical devices [8]. But applications where this kind of modelling strategy is competitive with finite element method are not so common. An innovation of the paper is to present an original application where this integral formulation is particularly efficient. Moreover, while the computation of ships magnetic anomaly can easily be achieved with FEM, currently the computation of coils effects is still unfeasible with this method on real ship geometries. The integral approach, with the presented dedicated implementation, seems to be very promising in order to model such coils effects and according to us remains certainly the only solution to model a real ship with its whole degaussing system.

In the second part of the paper, we will present the integral formulation used in this work, then we will quickly introduce the AMLFMM. Finally, we will compare modelling results obtained with measurements realized on a real submarine mock-up.

\section{INTEGRAL VOLUME EQUATION FORMULATION}

Let us consider a ferromagnetic shell placed in an inductor field $\mathbf{H}_{0}$ created by Earth and degaussing coils. The magnetic 
field is the sum of two terms: The inductor field $\mathbf{H}_{\mathbf{0}}$ and the demagnetization field $\mathbf{H}_{\text {red }}$ due to the magnetic reaction of the material

$$
\mathbf{H}=\mathbf{H}_{\mathbf{0}}+\mathbf{H}_{\text {red}} .
$$

In general, inductor field is very low in our application (order of magnitude of the Earth's magnetic field), so a linear magnetic behavior law is suitable to properly model the phenomenon. We have

$$
\mathbf{B}=\mu_{0} \mu_{r} \mathbf{H}
$$

where $\mu_{\mathrm{r}}$ is the constant, reduced, reversible and linear magnetic permeability of the material. It should be noticed that this reversible permeability has a low value (typically around 100) in comparison with anhysteric permeability classically used to model classical electrical system. This reversible permeability corresponds to small and linear variations of the magnetization when the material underlies such low fields. Considering that

$$
\mathbf{B}=\mu_{0}(\mathbf{H}+\mathbf{M})
$$

and combining it with (1) and (2), we get the expression of the induced magnetization $\mathbf{M}$

$$
\mathbf{M}=\left(\mu_{r}-1\right)\left(\mathbf{H}_{\mathbf{0}}+\mathbf{H}_{\text {red }}\right)
$$

where $\mathbf{H}_{\text {red }}$ is the field created by the whole magnetized shell and can be expressed with an integral equation. Combining this equation with (4), we obtain the classical integral volume equation for magnetostatic linear problem [8].

$$
\mathbf{M}=\left(\mu_{r}-1\right)\left(\mathbf{H}_{\mathbf{0}}+\frac{1}{4 \pi} \int_{V} \frac{3(\mathbf{M} . \mathbf{r}) \mathbf{r}-r^{2} \mathbf{M}}{r^{5}} d V\right)
$$

where $\mathrm{V}$ is the volume of the ferromagnetic material, $\mathbf{r}$ is the vector between a point of the shell and the integration point. Assuming the thickness $e$ of the hull is small and its $\mu_{\mathrm{r}}$ high, magnetization can be considered as locally tangential and constant through it [2]. Thus, (5) becomes

$$
\mathbf{M}=\left(\mu_{r}-1\right)\left(\mathbf{H}_{\mathbf{0}}+\frac{e}{4 \pi} \int_{S} \frac{3(\mathbf{M} . \mathbf{r}) \mathbf{r}-r^{2} \mathbf{M}}{r^{5}} d S\right)
$$

It remains to solve this integral equation. A very simple way is to consider 0-order shape functions for the magnetization. A point matching approach at barycenter of each element leads to $2 \mathrm{~N}$ equations (associated with 2 unknowns per element) where $\mathrm{N}$ is the number of surface elements

$\mathbf{M}_{j}=\left(\mu_{r}-1\right)\left(\mathbf{H}_{\mathbf{0}}-\frac{1}{4 \pi} \sum_{i}^{N} e_{i} \int_{S_{i}} \frac{3\left(\mathbf{M}_{i} \cdot \mathbf{r}\right) \mathbf{r}-r^{2} \mathbf{M}_{i}}{r^{5}} d S_{i}\right)$

where $\mathbf{M}_{\mathbf{j}}$ is the unknown magnetizations of element $\mathbf{j}$ (two components per element). To reduce the singularity for $i=j$ in (7), we used the well-known equivalent surface charge distribution of uniform magnetization to get

$$
\mathbf{M}_{j}=\left(\mu_{r}-1\right)\left(\mathbf{H}_{\mathbf{0}}-\frac{e}{4 \pi} \sum_{i}^{N} \int_{L_{i}} \mathbf{M}_{i} \cdot \mathbf{n}_{i} \frac{\mathbf{r}}{r^{3}} d L_{i}\right)
$$

where $\mathbf{n}$ is the external normal to each element tangential to the shell. Let's notice that in this formulation, sources are located on edges of the mesh and are equivalent to magnetic charge line distribution M.n. Thus, we get a full square matrix system of $2 \mathrm{~N}$ equations

$$
\left[\mathbf{I}_{\mathbf{d}}-\mathbf{G}\right][\mathbf{M}]=\left[\mathbf{h}_{\mathbf{0}}\right] .
$$

Remark: Equation (5) has been solved with a very basic approach and more sophisticated numerical methods can be applied (for instance, scalar potential formulation combined with a Galerkin projection of the equations). Moreover, high permeability materials can induce inaccuracies with this approach. However, for our application, values of order 100 are suitable for $\mu_{\mathrm{r}}$ because the inductor field is low enough. With such permeability, this formulation ensures good robustness and accuracy. Moreover, the 0-order description of the mesh allows the use of non-conformal mesh in a very simple way. This point is particularly important and facilitates the uneasy transfer of geometrical data (standard CAD files coming form mechanical description) to a meshed description of the ship.

System (9) (let us call it $\mathbf{A x}=\mathbf{b}$ ) can be solved with a direct solver (LU-decomposition) with a $\mathrm{O}\left(\mathrm{N}^{3}\right)$ complexity. A preconditioned iterative solver like GMRES reduces the computational costs to approximately $\mathrm{O}\left(\mathrm{N}^{2}\right)$. However, in most cases, the problem size is limited by the needed memory for the fully dense matrix storage $\left(\mathrm{O}\left(\mathrm{N}^{2}\right)\right)$. It is then vain to try to apply this method as it, to real ship geometries.

\section{AdAPTATiVe Multi-LeVel Fast Multipole Algorithm}

The FMM developed by Greengard and Rokhlin [9] has been successfully used to efficiently compute high number of potentials created by $\mathrm{N}$-charges problem. This hierarchical algorithm reduces the memory requirements and the computational cost of matrix-vector product in an iterative solver from $\mathrm{O}\left(\mathrm{N}^{2}\right)$ to approximately $\mathrm{O}(\mathrm{N} \log \mathrm{N})$. It keeps a known accuracy bounded by rigorously derived error bounds. With such algorithms, very large problems can be solved keeping an acceptable integration time and memory consumption. The classical version of this algorithm, based on spherical harmonic decompositions and a hierarchical octree partition of the domain, enables the computation of either potentials or fields in numerous points created by an important number of punctual charges without storing explicitly the interaction matrix.

\section{A. Magnetic Charges Computation}

In our approach, numerical integration of (8) by Gauss points technique leads to the introduction of punctual charges on edges of each element of the mesh. The charges values at each point are equal to the scalar product between the magnetization $\mathbf{M}$ and the external normal of element $\mathbf{n}$ multiplied by the weight of Gauss and the thickness of the shell

$$
\mathrm{q}=\mathrm{e} \times(\mathbf{M} . \mathbf{n}) .
$$

It should be noticed that in our formulation, the field is computed at each element barycenters so sources and points where the field are expressed are never the same (see Fig. 1). This numerical trick makes the computation of singular integrals more simple by reducing their singularity. Once we get all charges, 


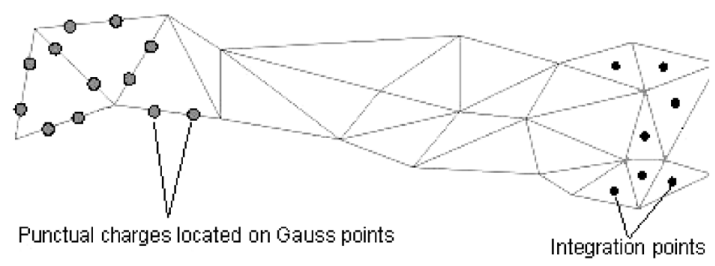

Fig. 1. Location of point where the field is expressed and punctual source charges on Gauss points.

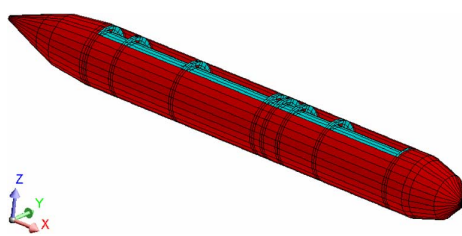

Fig. 2. The double hulls submarine mock-up.

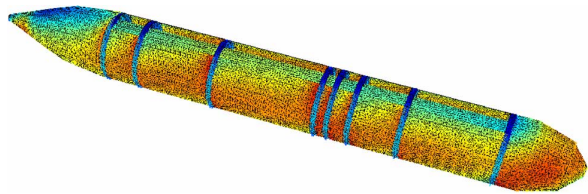

Fig. 3. Modulus of the induced magnetization created the earth magnetic field. During the solving process, 47 matrix-vector products are needed to obtain the final solution in 1800 seconds.

the classical approach FMM can easily be applied to the magnetic field computation.

\section{B. Fast Multipole Algorithm in GMRES}

The resolution of system of equations get by FMM must be obtained with an iterative solver, in our case GMRES. This method approximates the solution by a linear combination of Kyrlov subspace in order to minimize the residual $\mathbf{r}=\mathbf{b}-\mathbf{A x}$. To compute the matrix-vector product required in each GMRES iteration, we decompose the matrix into a near-field component and a far-field one. Thus, the matrix-vector product may be written

$$
\mathbf{A x}=\mathbf{A}^{\text {near }} \mathbf{x}_{\text {near }}+\mathbf{A}^{\text {far }} \mathbf{x}_{\mathbf{f a r}} .
$$

The near-field matrix represents the interactions between elements that are close to each other in the octree (elements which are in the same finest octree cube and elements belonging to neighbor cubes). Matrix $\mathbf{A}^{\text {near }}$ is built up explicitly and this matrix is stored. For the far-field product, the MLFM algorithm is used at each iteration step.

\section{Block-Diagonal Preconditioning}

One of the key points of the resolution is the convergence of the GMRES algorithm. The well-known technique of preconditioning is used in order to improve the convergence of the solver so as to reduce iterations' number. Thus, we have classically to modify the original system to get a new one with better condition number

$$
\mathbf{M}^{-1} \mathbf{A x}=\mathbf{M}^{-1} \mathbf{b}
$$
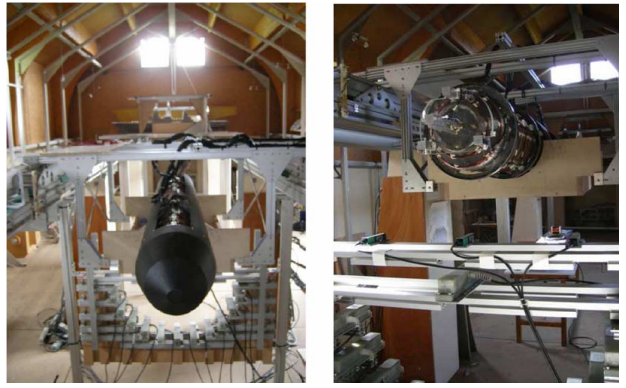

Fig. 4. Submarine Mock-up placed on the railway in the LMMCF. On the left, the mock-up in the simulator. On the right, only internal hull is shown with degaussing coils and fixed external fluxgate sensors.

where $\mathbf{M}$ is called the preconditioner matrix. Matrix $\mathbf{M}^{\mathbf{- 1}}$ should be as similar as possible to $\mathbf{A}^{\mathbf{- 1}}$, to significantly improve the condition number and speed-up the convergence. However, to build a preconditioner compatible with the FMM is somewhat different that with an explicit matrix solver, because many of the matrix elements are no longer explicitly available. Therefore, the preconditioner has to be built from the near-matrix elements only. In our work, we used a block diagonal preconditioner.

The explicit matrix $\mathbf{A}^{\text {near }}$ can be rewritten as in (13)

$$
\mathbf{A}^{\text {near }} \mathbf{x}_{\text {near }}=\mathbf{A}_{\mathbf{0}} \mathbf{x}_{\mathbf{0}}+\mathbf{A}_{\text {neighbor }} \mathbf{x}_{\text {neighbor }}
$$

where matrices $\mathbf{A}_{\mathbf{0}}$ represents the interaction matrix between each elements of a terminal cube and $\mathbf{A}_{\text {neighbor }}$ is the matrix interaction between these elements and elements belonging to neighbor terminal cubes. Let us notice that $\mathbf{A}_{0}$ is a square fully dense matrix but of a size managed by the adaptative octree.

For each terminal cube, the inverse matrix of each block $\mathbf{A}_{\mathbf{0}}$ is computed independently (this task is very rapid to complete, the size of the matrices being small and ensure an efficient preconditioning). These matrices are used as preconditioner.

\section{NumERICAL AND EXPERIMENTAL RESUlts}

Here, we focus on the specific double hull submarine structure: the internal one is dedicated to the pressure effects and external one is mainly hydrodynamic. Both hulls are made of ferromagnetic materials. A mock-up with realistic geometry has been built by DCNS company. Both hulls are separated by a 4 cm gap (Fig. 2):

- Internal hull (Thickness: $3 \mathrm{~mm}, \emptyset=30 \mathrm{~cm}$, length: $3 \mathrm{~m}$, $\left.\mu_{\mathrm{r}}=107\right)$;

—External hull (Thickness: $1 \mathrm{~mm}, \emptyset=38 \mathrm{~cm}$, length: 3.45 $\mathrm{m}, \mu_{\mathrm{r}}=180$ ).

A degaussing coils system has been installed. Coils are located between the two hulls and fixed on the internal one. It is composed of ten coils (seven longitudinal and three vertical). The mock-up is represented on Fig. 2.

Presented submarine example has been computed on a Dell PowerEdge 1950 with a multicore Intel(R) Xeon(R) CPU 5160 @ $3.00 \mathrm{GHz}$. Matrix-vector product is computed by a parallel version of AMLFMM. An original aspect of our work is that this algorithm has been developed in Java language. The submarine's hull is discretized with about 50,000 elements $(100,000$ 

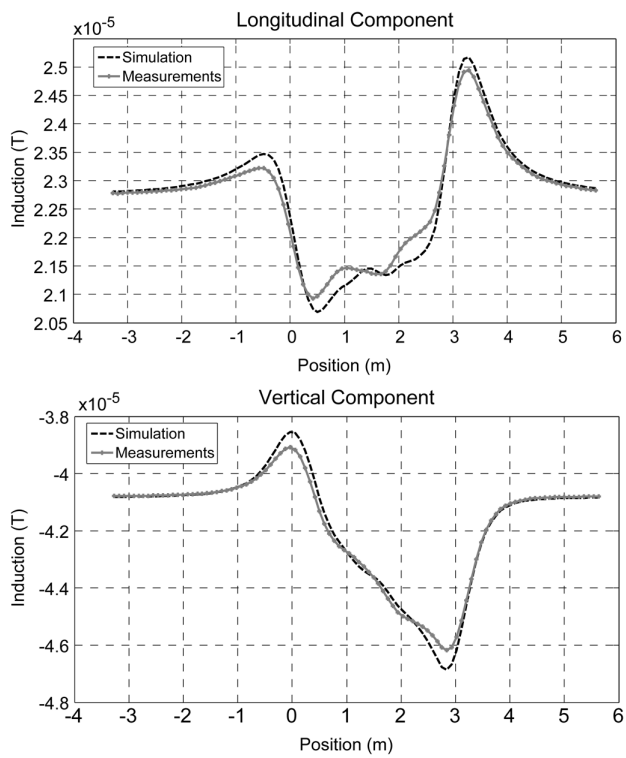

Fig. 5. Comparison between computed and measured signature for the mock-up placed in the Earth's magnetic field (longitudinal and vertical component of the magnetic field). The field is expressed $34 \mathrm{~cm}$ under the keel.

unknowns). The storage of the full system matrix would require more than $80 \mathrm{~GB}$. The use of the AMLFMM compression reduces the memory requirement to 2 GB (octree level $n=6$, multipole expansions truncations $\mathrm{p}=3$, Krilov subspace size $\mathrm{s}=200)$. The problem has been solved in 3 minutes.

Several modelings are proposed. The first one deals with the submarine in the Earth's magnetic field (the front of the mock-up in the north direction). The second one aims to compute a longitudinal coil effect. The computed magnetization obtained in the first modelling can be visualized in Fig. 3.

The submarine mock-up has been placed on a railway in a field simulator in the LMMCF (low magnetic field facility). This building is composed of several large coils enabling the control of surrounding field in a large space. This device allows also the cancellation of the Earth's field around the mock-up. Below the mock-up, several fixed fluxgate sensors have been placed, enabling the measurement of the whole magnetic anomaly on a line above the mock-up when it moves on the railway (see Fig. 4). Experimental results are compared with computations showing a very good adequacy for both scenarios (see Fig. 5 for the mock-up in the Earth's magnetic field and Fig. 6 for the degaussing coil effect) if we consider the complexity of the measurement process and the difficulty to properly determine right geometry and permeabilities.

\section{CONCLUSIONS}

In this paper, we have proposed to solve integral volume equation combined with a multi-level fast multipole method to model ship magnetic anomaly. This approach seems to be a very interesting alternative to the Finite Element Method traditionally
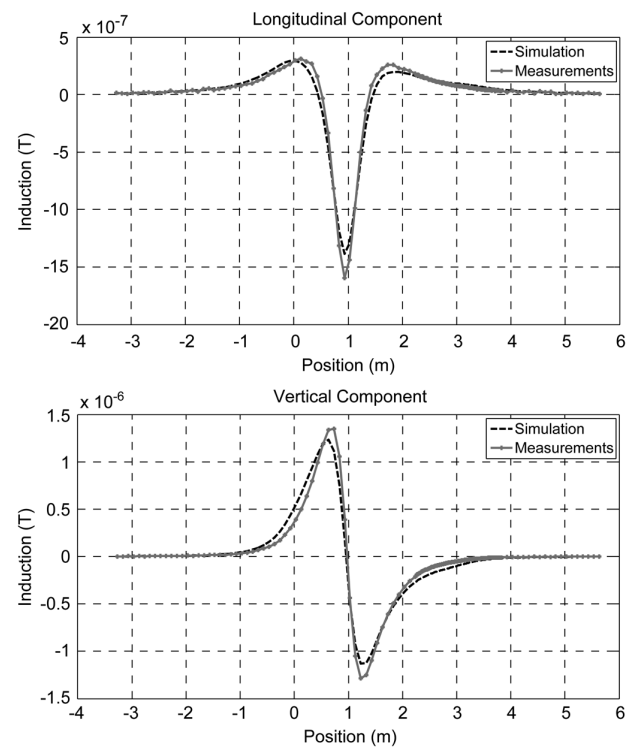

Fig. 6. Comparison between computed and measured signature for $1 \mathrm{~A}$ current in the third degaussing coil (longitudinal and vertical component of the magnetic field). The field is expressed $34 \mathrm{~cm}$ under the keel.

used. The method has shown a very good fit and enables the resolution of problems with very dense surface meshes. Moreover, the approach has demonstrated its efficiency in solving degaussing coils effects problem keeping acceptable memory and computation times.

\section{REFERENCES}

[1] X. Brunotte, G. Meunier, and J. F. Imhoff, "Finite elements solution of unbounded problems using transformations: A rigorous, powerful and easy solution," IEEE Trans. Magn., vol. 28, pp. 1663-1666, 1992.

[2] X. Brunotte and G. Meunier, "Line element for efficient computation of the magnetic field created by thin iron plates," IEEE Trans. Magn., vol. 26, pp. 2196-2198, 1990.

[3] F. Le Dorze, J.-P. Bongiraud, J.-L. Coulomb, P. Labie, and P. X. Brunotte, "Modeling of degaussing coils effects in ships by the method of reduced scalar potential jump," IEEE Trans. Magn., vol. 34, pp. 2477-2480, 1998.

[4] O. Chadebec, J.-L. Coulomb, V. Leconte, J. P. Bongiraud, and G. Cauffet, "Modeling of static magnetic anomaly created by iron plates," IEEE Trans. Magn., vol. 36, pp. 667-671, Jul. 2000.

[5] K. J. Lee, G. Jeung, C.-S. Yang, H.-J. Chung, J. G. Park, H.-G. Kim, and D.-H. Kim, "Implementation of material sensitivity analysis for determining unknown remanent magnetization of a ferromagnetic thin shell," IEEE Trans. Magn., vol. 45, pp. 1478-1481, 2009.

[6] G. Jeung, C.-S. Yang, H.-J. Chung, S.-H. Lee, and D.-H. Kim, "Magnetic dipole modeling combined with material sensitivity analysis for solving an inverse problem of thin ferromagnetic sheet," IEEE Trans. Magn., vol. 45, pp. 4169-4172, 2009.

[7] O. Chadebec, J.-L. Coulomb, L.-L. Rouve, J.-P. Bongiraud, G. Cauffet, and P. Le Thiec, "Recent improvements for solving inverse magnetostatic problem applied to thin shells," IEEE Trans. Magn., vol. 38, pp. 1005-1008, 2002.

[8] W. Hafla, F. Groh, A. Buchau, and W. M. Rucker, "Magnetic field computation with integral equation method and energy-controlled relaxation," IEEE Trans. Magn, vol. 42, pp. 719-722, 2006.

[9] J. Carrier, L. Greengard, and V. Rokhlin, "A fast adaptive multipole algorithm for particle simulation," SIAM, J. Sci. Statist. Comput., vol. 9, p. 669,1988 